\title{
OPTIMAL INTEGRATION-FORMULAS FOR ANALYTIC FUNCTIONS
}

\author{
BY R. B. BARRAR, ${ }^{1}$ H. L. LOEB, ${ }^{2}$ AND H. WERNER
}

Communicated by Eugene Isaacson, April 13, 1973

We prove the existence of best approximate quadrature formulas for evaluating integrals that involve analytic functions. In [1] Eckhardt and in [2], [3], [4] Richter-Dyn have considered optimal integration formulas for certain classes of Hilbert spaces of analytic functions defined as follows.

Let $S$ be a simply connected domain in the complex plane symmetric about the real axis. Further let $\phi(z)$ be a conformal mapping from $S$ onto the unit disk with the property that for some real $z_{0} \in S$,

$$
\phi\left(z_{0}\right)=0, \quad \phi^{\prime}\left(z_{0}\right)>0 .
$$

Let $[\alpha, \beta]$ be a real interval in $S$ and $\rho(z)$ be a function defined on $S$ so that both $\rho(z)$ and $1 / \rho(z)$ are analytic in $S$ and $\rho(z)$ is strictly positive for real $z$ in $S$.

$H^{2}(S, \rho)$ is the Hilbert space of functions $f$ analytic in $S$ and satisfying

$$
\int_{\partial S}|f(z)|^{2}|\rho(z)|^{2}|d z|<\infty,
$$

where $\partial S$ is the boundary of $S$. This space has the inner-product

$$
(f, g)=\int_{\partial S} f(z) \bar{g}(z)|\rho(z)|^{2}|d z|,
$$

and the reproducing kernel $[5$, p. 79$]$

$$
K(x, y)=\frac{1}{2 \pi} \frac{\left(\phi^{\prime}(x) \overline{\left.\phi^{\prime}(y)\right)^{1 / 2}}\right.}{\rho(x) \overline{\rho(y)}} \cdot[1-\phi(x) \overline{\phi(y)}]^{-1}
$$

For this space and a fixed positive integer $n$ Richter-Dyn considers quadrature formulas of the type $\sum_{i=1}^{n} a_{i} f\left(y_{i}\right)$, where $a_{i}, y_{i}$ are real and $y_{i}$

AMS (MOS) subject classifications (1970). Primary 41A50, 41A30; Secondary 41A55, 30A82.

${ }^{1}$ Supported in part by NSF Grant GP-28111.

${ }^{2}$ Supported in part by Sonderforschungsbereich 72 at the Institute for Applied Mathematics, University of Bonn, and NSF Grant GP-18609. 
is in $S(i=1, \ldots, n)$, to approximate

$$
L(f)=\int_{\alpha}^{\beta} f(x) w(x) d x
$$

where $w(x)$ is a fixed strictly positive continuous function and $f(x)$ is an arbitrary element of the Hilbert space. Further Richter-Dyn seeks the optimal quadrature formula [3]; that is find the $\left\{a_{i}\right\},\left\{y_{i}\right\}$ which minimize

$$
\left\|\phi(x)-\sum_{i=1}^{n} a_{i} K\left(x, y_{i}\right)\right\|,
$$

where $\phi(x)$ is the representer of $L(f)$; that is $\phi(x)=\int_{\alpha}^{\beta} K(x, y) w(y) d y$. Here $\|\cdot\|$ is the Hilbert space norm.

Richter-Dyn shows any best approximation is of the form

$$
\sum_{i=1}^{n} a_{i} K\left(x, y_{i}\right)
$$

where $a_{i}>0, y_{i} \in(\alpha, \beta)(i=1, \ldots, n), y_{i} \neq y_{j}$, but does not show that a best approximation exists.

Attempts of proofs in [1], [2] fail to consider the behaviour of a functional at the points $y_{i}(i=1, \ldots, n)$ if two or more of the $y_{i}$ coincide. This deficiency has already been pointed out by Richter-Dyn [3].

We can consider a more general class of quadrature formulas; that is formulas of the type,

$$
\sum_{i=1}^{t} \sum_{j=0}^{m_{i}-1} a_{i j} f^{(j)}\left(y_{i}\right)+\sum_{i=1}^{t^{\prime}} \sum_{j=0}^{m_{i}^{\prime}-1}\left[b_{i j} f^{(j)}\left(y_{i}^{\prime}\right)+\bar{b}_{i j} f^{(j)}\left(\bar{y}_{i}^{\prime}\right)\right]
$$

Here, $a_{i j}$ real, $y_{i}$ real and is in $S, b_{i j}$ complex, $y_{i}^{\prime}$ complex and is in $S$, and

$$
\sum_{i=1}^{t} m_{i}+2 \sum_{i=1}^{t^{\prime}} m_{i}^{\prime} \leqq n .
$$

A special case of our theory is that for the class (3) a best approximation does exist in the sense of (1). Further any best approximation is of the form (2). Our main tools in proving these results are some results in the theory of moments $[6, \mathrm{pp} .45,46]$ and the concept of extended varisolvence [7]. For some related results one is referred to [8], [9].

\section{REFERENCES}

1. U. Eckhardt, Einige Eigenschaften Wilfscher Quadraturformeln, Numer. Math. 12 (1968), 1-7. MR 41 \# 1217.

2. N. Richter, Properties of minimal integration rules, SIAM J. Numer. Anal. 7 (1970), 67-79. MR 41 \# 4804.

3. N. Richter-Dyn, Properties of minimal integration rules. II, SIAM J. Numer. Anal. 8 (1971), 497-508. 
4. N. Richter-Dyn, Minimal interpolation and approximation in Hilbert spaces, SIAM J. Numer. Anal. 8 (1971), 583-597.

5. S. Bergman, The kernel functions and conformal mapping, Math. Surveys, no. 5, Amer. Math. Soc., Providence, R.I., 1950. MR 12, 402.

6. S. Karlin and W. S. Studden, Tchebycheff-systems: With applications in analysis and statistics, Pure and Appl. Math., vol. 15, Interscience, New York, 1966. MR 34 \# 4757.

7. R. B. Barrar and H. L. Loeb, On extended varisolvent families, J. Analyse Math. (to appear).

8. S. Karlin, On a class of nonlinear approximation problems, Bull. Amer. Math. Soc. 78 (1972), 43-49. MR 45 \# 786.

9. R. B. Barrar and H. L. Loeb, Analytic extended monosplines (submitted for publication).

Department of Mathematics, University of Oregon, Eugene, Oregon 97403

Gesellschaft für Mathematik und Datenverarbeitung mbH Bonn, St. AugustinBirlinghoven, Federal Republic of Germany 